\title{
Mayo Clinic Recognized by Three Certifying Boards for Quality Improvement Activities
}

The American Board of Family Medicine (ABFM), the American Board of Internal Medicine (ABIM), and the American Board of Pediatrics ( $\mathrm{ABP}$ )— the nation's 3 largest physician certifying boards-announced that Mayo Clinic has been approved as a Portfolio Sponsor of Maintenance of Certification (MOC) activities. Mayo Clinic's inclusion in the pilot MOC Portfolio Approval Program was based on the boards' recognition of Mayo Clinic's rigorous attention to detail and the structure of its physician quality improvement (QI) programs.

As an MOC Portfolio Sponsor, Mayo Clinic has primary ownership of the design and execution of a large number of QI activities that are managed centrally through an established infrastructure and overseen by a formal governance body. Mayo Clinic will also accept accountability for ensuring the activities meet the standards outlined by the 3 boards and for management of the QI activities.

Although the ABFM, the ABIM, and the ABP have individually recognized other organizations' QI products and programs for MOC credit in the past, Mayo Clinic is the first organization to be recognized, jointly, by all 3 boards. The 3 primary care boards expect to approve 2 to 4 additional portfolio sponsor organizations in the next 3 years as part of this effort.

"We are proud that Mayo Clinic is the first organization recognized by the 3 largest certifying boards and believe that this is a testament to Mayo's leadership in quality improvement," said Richard Berger, MD, Dean of the Mayo School of Continuous Professional Development. "We believe rigorous quality improvement efforts make better physicians, improve the systems of care that physicians work in, and, most importantly, enhance patient care, furthering Mayo Clinic's leadership in the practice of medicine. We are delighted that family physicians, internists, and pediatricians who are engaged in QI activities every day will receive MOC credit for their hard work."
Research has shown that fewer than $30 \%$ of physicians examine their own performance data, and physicians' ability to independently self-assess and self-evaluate is poor. Each certifying board requires physicians to look at their practice and make improvements. Mayo Clinic has established QI activities in its clinical setting that meet the 3 primary care boards' requirements for improving performance in practice.

"We look forward to recognizing other programs that engage physicians in rigorous quality improvement activities," said James A. Stockman, MD, President and CEO of the American Board of Pediatrics. "Physicians want to deliver the best care possible to their patients, and these programs help them understand where improvement is needed and give them a structured environment to make positive changes."

Mayo Clinic's Quality Review Board will evaluate Mayo physicians' participation in structured QI activities to determine whether they meet the boards' requirements for MOC. Among the requirements for MOC approval:

- Projects must focus on one of the Institute of Medicine's (IOM) 6 dimensions of quality (ie, making health care more safe, effective, patientcentered, timely, efficient, and equitable).

- Physicians must provide direct or consultative care to patients as part of the QI project or actively participate in the process of care being addressed by the project. This includes physicians who actively supervise a trainee (resident or fellow) during a QI project.

- Physicians must demonstrate active collaboration in QI project design and/or implementation, such as team meetings, data analysis, implementation training, etc.

"MOC is a multifaceted program that includes knowledge and continuous improvement," said Christine Cassel, MD, President and CEO of the American Board of Internal Medicine. "We look for opportunities to reduce the administrative bur- 
den for physicians, but still ensure that they are meeting our high standards of self-evaluation and quality improvement."

"The 3 primary care boards are working together to develop novel programs that can be used to maintain certification, and this collaborative pilot with Mayo Clinic represents just one of our innovative projects," said James C. Puffer, MD, President and CEO of the American Board of Fam- ily Medicine. "We continue to explore next generation approaches to quality improvement, including those that interface with integrated health care systems, community-based medical groups, and the individual physician's practice."

Kevin Graves American Board of Family Medicine American Board of Internal Medicine American Board of Pediatrics 